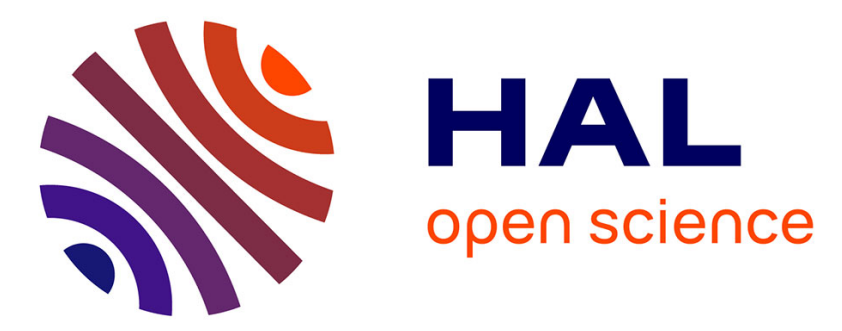

\title{
LA NOUVELLE CLASSIFICATION DIAGNOSTIQUE DE LA PETITE ENFANCE (DC: 0-5) : CONTINUITÉS ET ÉVOLUTIONS
}

Patrick Perret, Julie Le Foll, Antoine Guedeney

\section{- To cite this version:}

Patrick Perret, Julie Le Foll, Antoine Guedeney. LA NOUVELLE CLASSIFICATION DIAGNOSTIQUE DE LA PETITE ENFANCE (DC: 0-5) : CONTINUITÉS ET ÉVOLUTIONS. Devenir - Revue européenne du développement de l'enfant, 2019, 10.3917/dev.193.0197 . hal-02468664

\section{HAL Id: hal-02468664 \\ https://hal.science/hal-02468664}

Submitted on 7 Feb 2020

HAL is a multi-disciplinary open access archive for the deposit and dissemination of scientific research documents, whether they are published or not. The documents may come from teaching and research institutions in France or abroad, or from public or private research centers.
L'archive ouverte pluridisciplinaire HAL, est destinée au dépôt et à la diffusion de documents scientifiques de niveau recherche, publiés ou non, émanant des établissements d'enseignement et de recherche français ou étrangers, des laboratoires publics ou privés. 


\title{
La nouvelle classification diagnostique de la petite enfance DC :0-5 : continuités et évolutions
}

\author{
Patrick PERRET \\ Julie Le Foll et Antoine GUEDENEY \\ Patrick Perret est psychologue, maître de conférences en psychologie. Il a traduit en français la DC0-3 R et la DC 0-5. \\ Aix-Marseille Univ, PsyCLE, Aix-en-Provence, France. \\ Julie Le Foll est praticien hospitalier, policlinique Ney Jenny Aubry, Hôpital Bichat Claude Bernard. Paris 75018, \\ Antoine Guedeney est pédopsychiatre, professeur de psychiatre de l'enfant et de l'adolescent, et chef de policlinique Ney \\ Jenny Aubry, Paris 75018 Hôpital Bichat Claude Bernard. GHPVS, et Paris 7, Paris. \\ Auteur correspondant : Patrick Perret : E-mail : Patrick.Perret@univ-amu.fr
}

Reçu novembre 2018, révisé et accepté janvier 2019

\section{Résumé :}

La classification diagnostique de la santé mentale et des troubles du développement de la petite enfance (DC:0-5) a été publiée en 2016 par I'organisation ZERO TO THREE aux Ełats-Unis, sous la direction d'un groupe de travail dirigé par Charles Zeanah. Elle constitue la version révisée et actualisée d'une classification spécifiquement dédiée aux troubles de la petite enfance (DC:0-3, 1994) impulsée par Stanley Greenspan et révisée une première fois en 2005 (DC :0$3 R$ ) par un groupe de travail sous la direction de Robert Emde. La nouvelle version de cette classification, aujourd'hui publiée en langue française, préserve plusieurs principes fondateurs des versions précédentes mais elle introduit également quelques évolutions substantielles. L'objectif du présent article est d'aider les futurs utilisateurs francophones de cet instrument diagnostique à en repérer la philosophie générale, la continuité et les changements.

\section{Abstract :}

The Diagnostic Classification of Mental Health and Developmental Disorders of Infancy and Early Childhood (DC:0-5TM) was published in 2016 by the organization ZERO TO THREE in the United States, under the direction of a Task Force chaired by Charles Zeanah. It constitutes the revised and updated version of a diagnostic 
classification specifically dedicated to early childhood disorders (DC: 0-3, 1994) promoted by Stanley Greenspan, and revised a first time in 2005 (DC: 0-3R) by a previous revision task force, chaired by Robert Emde. The new edition of this classification, now published in French, preserves several founding principles of previous versions, but it also introduces some substantial developments. The aim of the present paper is to help future French speaking users of this diagnostic instrument to identify its general philosophy, continuity and changes. 


\section{La nouvelle classification diagnostique de la petite enfance DC:0-5 : continuités et évolutions}

En 2016 est parue aux États-Unis la nouvelle édition de la Classification Diagnostique de la santé mentale et des troubles du développement de la petite enfance ( $D C: 0-5^{\mathrm{TM}}$ ). Elle constitue la version actualisée et révisée de la classification DC :0-3R, publiée en français en 2009 dans la revue Devenir. Cette nouvelle édition, dont la traduction française paraît aujourd'hui, préserve certains aspects fondamentaux du système diagnostique mais introduit également des modifications nourries par les progrès de la recherche dans le domaine de la santé mentale du jeune enfant et des retours d'expérience clinique. Le texte qui suit vise à aider les utilisateurs de l'ancienne édition à repérer ces changements et à aider les nouveaux utilisateurs à comprendre la logique d'ensemble et l'utilité clinique de cet instrument.

\section{De l'usage des classifications diagnostiques}

L'usage des classifications diagnostiques dans le domaine de la santé mentale fait l'objet de débats récurrents chez les chercheurs et les cliniciens. II convient donc, avant d'examiner les évolutions qui caractérisent ce nouvel instrument diagnostique, d'en repréciser la philosophie générale.

Tout clinicien confronté à la situation d'un enfant présentant des difficultés psychologiques s'engage nécessairement dans un processus intellectuel consistant à rapprocher cette situation singulière de tableaux cliniques que son expérience professionnelle ou sa connaissance de la littérature l'ont conduit à élaborer. Ce rapprochement peut être implicite ou bien, lorsqu'il s'opère sur la base d'une classification diagnostique, prendre la forme d'une réflexion explicite, systématique et partageable. Les classifications offrent alors un cadre permettant d'une part de systématiser ce processus de rapprochement par l'intermédiaire de critères établis, d'autre part de communiquer sur les situations cliniques par l'intermédiaire de concepts partagés : "L'objectif premier de cette activité de classification est que les professionnels - cliniciens, chercheurs, responsables médico-sociaux - puissent communiquer efficacement à propos de syndromes descriptifs ॥ (DC:0-5, 2016, p. 7). Pour autant, l'utilisation d'un concept diagnostique pour décrire de façon synthétique un pattern de symptômes n'épuise pas le processus de compréhension clinique et ce, pour au moins trois raisons. D'abord parce que le fonctionnement psychologique d'un enfant et la dynamique de son développement ne se réduisent 
naturellement pas à l'organisation des symptômes susceptibles d'être observés. Ensuite parce que la description synthétique de ces symptômes ne constitue pas en soi leur explication: "Nous avons abordé ce travail de façon a-théorique, avec l'objectif de fournir des critères descriptifs qui se prêtent à l'évaluation de leur fidélité et de leur validité, en minimisant le recours à des inférences ou des attributions qui excéderaient ce qui est cliniquement observable ॥ (DC:0-5, 2016, p. 1.). Enfin parce que la réflexion diagnostique dans le domaine complexe des troubles mentaux a vocation à prendre la forme d'un processus critique et continu : "Un diagnostic n'est pas formulé sur la base d'un instantané de symptômes mais sur une collecte continue d'informations sur le fonctionnement du jeune enfant pour comprendre les multiples aspects de ses difficultés et les variations d'adaptation et de développement qui se manifestent à différentes occasions et dans différents contextes ॥ (DC:0-5, 2016, p. 7).

L'élaboration de la classification elle-même a pris la forme d'un processus collégial d'une durée de trois années. Plusieurs sections de son introduction rappellent les démarches successivement engagées pour faire évolver le système diagnostique sur la base des retours d'expérience clinique, de la recension des travaux de recherches, et des commentaires d'experts sollicités à partir de versions préliminaires. La classification 0-5 ans ne constitue donc pas - au même titre que toutes les autres classifications - l'expression définitive de notre connaissance des troubles, mais une proposition dont peuvent se saisir les chercheurs et les cliniciens de la petite enfance pour les aider à organiser leurs pratiques de recherche, d'évaluation ou de soin.

\section{Continuités dans l'organisation et la finalité de la Classification}

L'une des raisons d'être de la classification 0-5 ans est que les difficultés psychologiques rencontrées par les jeunes enfants et leurs familles prennent des formes spécifiques durant les premières années de la vie. L'expérience clinique montre que les classifications diagnostiques générales comme les systèmes DSM ou CIM ne permettent pas d'appréhender suffisamment finement la diversité et la spécificité des troubles tels qu'ils peuvent se manifester à l'âge préscolaire. En outre, les auteurs insistent sur la nécessité d'aborder les difficultés de cette période avec une approche développementale. Elle implique une attention particulière pour les évolutions rapides comme pour les trajectoires qui se dessinent, la prise en compte de la variabilité individuelle induite par le tempérament et la personnalité en construction, la prise en compte du rythme habituel auquel les enfants élaborent leurs compétences dans les différents domaines du développement, et une attention particulière pour les environnements au sein desquels évoluent les jeunes enfants. Le développement individuel du jeune enfant est en effet profondément 
influencé, plus qu'à toute autre période de la vie, par les expériences relationnelles qui lui sont proposées: les relations humaines constituent son principal contexte de développement.

Dès lors il apparaît nécessaire, pour permettre aux professionnels d'élaborer une représentation de la situation clinique d'un jeune enfant, d'aller au-delà de l'expression symptomatique et d'examiner l'ensemble des facteurs qui concourent à la dynamique de son développement. C'est la raison pour laquelle la classification a résolument préservé au cours de ces différentes éditions une forme multiaxiale. Les cinq axes qui la composent invitent le clinicien à focaliser successivement son attention sur (1) les symptômes de l'enfant et leur correspondance avec des patterns connus, (2) la qualité des relations qu'il a élaboré avec ses parents et la façon dont ces relations soutiennent son développement, (3) sa situation médicale et son incidence éventuelle, (4) les facteurs de stress psychosociaux qui exercent des contraintes sur la vie de la famille, (5) le niveau actuel de fonctionnement du jeune enfant et les compétences manifestées dans les domaines du développement social, émotionnel, cognitif, psychomoteur, communicatif et langagier. Cette organisation multiaxiale est exigeante: examiner la situation clinique sous ces différents angles implique à la fois des rencontres multiples avec une famille et la coordination de plusieurs professionnels ayant des domaines d'expertise différents: "Dans cette perspective, un clinicien ou une équipe ont nécessairement besoin de plusieurs séances pour appréhender comment un bébé ou un jeune enfant se développe dans chaque domaine de fonctionnement. Quelques questions aux parents ou aux donneurs de soins peuvent permettre un premier tour d'horizon mais ne sauraient constituer à elles seules une évaluation. Une évaluation complète implique habituellement un minimum de trois à cinq séances de 45 minutes ou plus chacune" (DC:0-5, 2016, p. 8). Cette organisation est également précieuse en ce qu'elle fait de la classification DC:0-5 bien plus qu'un simple répertoire de troubles : elle constitue, pour les équipes de professionnels qui la mobilisent, un guide possible au service du processus d'élaboration clinique.

"La notion d'élaboration clinique désigne la façon dont le tableau clinique présenté par un jeune enfant est compris dans le contexte des relations qu'il a pu former, de ses caractéristiques biologiques, de son environnement social et de son niveau de développement. Ce processus d'élaboration oriente les stratégies d'intervention en mettant en lumière les facteurs de risque et les facteurs de protection, en identifiant ceux d'entre eux qui sont modifiables, et en établissant des priorités dans la prise en charge des facteurs cliniques» (DC:0-5, 2016, p. 7). Renseigner les différents axes de la classification DC:0-5 permet à une équipe d'organiser le recueil des informations et le processus d'évaluation. Ce faisant, le processus d'élaboration clinique conduit à mettre progressivement en lumière les différents registres de difficultés auxquelles un jeune enfant et sa famille sont 
confrontés mais également les ressources dont ils disposent. La représentation globale qui émerge de ce processus d'élaboration permet alors aux équipes d'envisager le niveau et les formes d'aide dont le jeune enfant pourrait bénéficier pour soutenir son développement.

\section{Evolution des différents axes}

Le lecteur pourra se reporter à Guedeney et Le Foll (sous presse) pour une recension exhaustive et une mise en perspective critique des différents changements intervenus entre les éditions DC:0-3R (2005) et DC:0-5 (2016). Dans les paragraphes qui suivent, nous attirons l'attention des utilisateurs de l'ancien système sur les changements les plus significatifs pour l'utilisation clinique de la classification.

\section{Axe 1 : Troubles cliniques}

L'Axe 1 est utilisé pour caractériser les patterns de symptômes observés dans le fonctionnement du jeune enfant lui-même. Une première évolution notable de cet axe tient à la période développementale considérée: alors que les précédentes éditions étaient focalisées sur un empan d'âge 0-3 ans, la nouvelle édition s'étend à la période préscolaire dans son ensemble, c'est-à-dire avant 6 ans. Une seconde évolution relève d'un effort d'exhaustivité plus soutenu dans l'identification des différents registres de troubles susceptibles d'être observés durant cette période et de mise en cohérence avec les systèmes nosographiques de la CIM-10 et du DSM-5.

Au sein des troubles neuro développementaux, deux tableaux cliniques nouveaux sont notamment proposés: le Trouble précoce atypique du spectre de l'autisme et le Trouble de suractivité de la petite enfance. Ces concepts décrivent des situations de très jeunes enfants (avant 3 ans) présentant une symptomatologie de type autistique ou de trouble attentionnel mais sans toutefois remplir l'ensemble des critères diagnostiques d'un Trouble du spectre de l'autisme ou de Déficit de l'attention/hyperactivité. Ces concepts nosographiques permettent alors de caractériser (sans préjuger de l'évolution de la trajectoire développementale) des tableaux sub-cliniques méritant de la part des professionnels de la petite enfance une attention particulièrement soutenue.

Deux autres tableaux également très spécifiques de la petite enfance sont désormais décrits: la Dysrégulation de la colère et de l'agressivité de la petite enfance et les Pleurs pathologiques. Ces deux tableaux permettent aux cliniciens de la petite enfance de conceptualiser des situations qu'ils rencontrent régulièrement, car les difficultés de régulation et de pleurs constituent de fréquents motifs de consultation. Ils illustrent toutefois avec acuité la difficulté générale à établir les seuils au-delà desquels un mode de fonctionnement chez l'enfant entre dans le registre d'un trouble. L'examen des algorithmes diagnostiques proposés par la classification indique que trois critères apparaissent systématiquement et sont donc 
consubstantiels du concept de «trouble». Un trouble désigne une situation clinique qui s'éloigne significativement des normes développementales habituelles dans un contexte culturel donné et concerne donc, par définition, une très faible proportion d'enfants. Un deuxième critère récurrent est le fait que les symptômes qui caractérisent le trouble se manifestent de façon envahissante, c'est-à-dire s'étendent à différents contextes et à différentes relations. Enfin, le caractère atypique du fonctionnement d'un enfant ne justifie jamais, à lui seul, d'être envisagé comme relevant d'un trouble: il ne le devient potentiellement que lorsque ce fonctionnement à la fois atypique et envahissant engendre chez le jeune enfant et sa famille des situations de souffrance et d'inadaptation qui altèrent sa dynamique de développement. Ces situations méritent d'être reconnues comme telles et appellent alors une démarche de soin.

Une exception au critère "envahissant" concerne toutefois le dernier trouble répertorié sous l'Axe 1 : le Trouble relationnel spécifique de la petite enfance. Ce concept diagnostique désigne l'apparition de symptômes chez l'enfant (quel qu'en soit le registre) uniquement lorsque ce dernier se trouve en relation avec une figure adulte spécifique. Dans les précédentes éditions de la classification, les troubles de la relation étaient décrits et conceptualisés sous l'Axe 2. Or, comme nous allons le voir, cet axe est désormais conçu pour décrire systématiquement le contexte relationnel dans lequel grandit un jeune enfant, et non plus uniquement les contextes troublés. Les situations cliniques caractérisées par un trouble de la relation qui engendrent une expression symptomatique chez l'enfant sont donc désormais identifiées sous l'Axe 1.

\section{Axe 2 : Contexte relationnel}

Comme nous l'indiquions plus haut, les relations que forme le jeune enfant avec des adultes de référence (ses « donneurs de soin », la plupart du temps ses parents) constituent son principal contexte de développement : les expériences relationnelles régulières qui lui sont proposées structurent peu à peu ses attentes sur la façon dont fonctionnent les relations humaines et soutiennent le développement progressif de ses capacités d'autorégulation dans les différents secteurs de son développement. L'Axe 2 invite les cliniciens de la petite enfance à un effort systématique pour explorer et caractériser ce contexte relationnel qui constitue, au même titre que les symptômes éventuellement présentés par l'enfant, un élément fondamental de sa situation clinique. L'évaluation du contexte relationnel sous l'Axe 2 repose désormais sur deux échelles : l'échelle de Niveaux d'adaptation de la relation parent-enfant et l'échelle de Niveaux d'adaptation de l'environnement familial.

La première échelle est utilisée pour caractériser le fonctionnement de la relation parent-enfant elle-même. Quatre niveaux généraux d'adaptation y sont 
décrits, du niveau 1 (Relation bien adaptée à satisfaisante) au niveau 4 (Relation troublée à dangereuse) et associés à différents degrés d'urgence d'intervention de la part des professionnels. La formulation de ce niveau global d'adaptation intervient au terme d'un processus d'exploration clinique de la relation. Cette exploration implique notamment une analyse des interactions comportementales parent-enfant dans différents contextes afin d'être représentative des expériences habituelles du jeune enfant. Elle implique également une analyse approfondie, par le biais d'entretiens cliniques, de la façon dont un parent se représente le fonctionnement de son enfant ainsi que la relation qu'ils élaborent. Afin de guider cette exploration du contexte relationnel sur laquelle se fonde la formulation du niveau d'adaptation, l'Axe 2 propose au clinicien de renseigner deux tableaux qui synthétisent la contribution respective du parent et de l'enfant au fonctionnement relationnel observé. Le tableau 1 aide à repérer les dimensions de la parentalité qui sont importantes à considérer dans l'analyse (comme la capacité du parent à manifester de l'intérêt pour les expériences de son enfant ou sa capacité à assurer la sécurité physique de l'enfant). Le tableau 2 recense les caractéristiques propres de l'enfant (comme son tempérament ou son profil sensoriel) qui contribuent elles aussi au fonctionnement de la relation en imposant au parent des formes d'ajustement plus ou moins exigeantes.

La seconde échelle est utilisée pour caractériser le fonctionnement de l'environnement familial dans son ensemble. Là encore, l'échelle comporte quatre niveaux d'adaptation, précédés d'un tableau de synthèse des dimensions du fonctionnement familial sur lesquelles l'évaluation peut se centrer, comme les modalités de répartition des rôles et de résolution des conflits ou l'harmonie entre les membres de la fratrie. En effet, indépendamment de la qualité des relations duelles parent-enfant, la fluidité de la communication entre les membres de la famille élargie et la convergence des systèmes de valeur et des pratiques éducatives participent à offrir au jeune enfant une cohérence d'expériences qui contribue à son développement.

\section{Axe 3 : État de santé physique du jeune enfant}

La situation pédiatrique générale du jeune enfant peut exercer, par l'intermédiaire de phénomènes de cascades développementales, des effets importants sur son développement psychologique, sur ses symptômes et sur ses relations. C'est le cas - par exemple - de syndromes génétiques affectant le développement de certaines aptitudes cognitives ou motrices, d'expériences de douleurs affectant le sommeil et l'alimentation, ou encore de maladies chroniques affectant la perception par les parents de la vulnérabilité de l'enfant. Par conséquent, l'Axe 3 invite les équipes à être attentives à ces éléments médicaux et 
à leurs incidences directes ou indirectes. Dans sa nouvelle édition, l'axe bénéficie d'une recension des informations médicales à considérer pour aider les cliniciens à organiser le processus de recueil des informations.

\section{Axe 4 : Facteurs de stress psycho-sociaux}

Si le développement du jeune enfant advient toujours dans le contexte des relations qu'il élabore avec ses donneurs de soin, ces relations elles-mêmes adviennent toujours dans un contexte social et culturel plus large qui exerce des contraintes sur le fonctionnement familial. L'Axe 4 invite les équipes à prendre en considération les facteurs de stress psycho-sociaux qui pèsent sur une famille et à réfléchir à leurs effets en gardant à l'esprit deux principes importants. Le premier est qu'un facteur de stress donné, comme un événement potentiellement traumatique, n'aura pas nécessairement le même effet chez tous les enfants car son incidence est médiatisée par d'autres variables comme le niveau de développement de l'enfant (qui en module les ressources adaptatives) ou la capacité des adultes à constituer un filtre protecteur (qui varie selon les contextes relationnels). Un second principe correspond au phénomène du risque cumulatif : des recherches empiriques signalent que le nombre de facteurs de risques cumulés par une famille est un prédicteur de difficultés pour le développement de l'enfant plus important que tel ou tel facteur considéré isolément.

Comme dans la précédente édition, l'Axe 4 propose donc un Inventaire des facteurs de stress psycho-sociaux permettant aux équipes de recenser les événements, situations, ou contraintes de différentes natures (ex. médicale, économique, éducative, judiciaire) susceptibles d'influencer le fonctionnement actuel de l'environnement familial.

\section{Axe 5 : Compétences développementales}

L'analyse de la situation clinique d'un jeune enfant requiert un examen attentif de son niveau de développement pour plusieurs raisons. De nombreux troubles définis sur l'Axe 1 impliquent dans leurs critères diagnostiques un retard dans la construction de compétences développementales spécifiques comme par exemple des aptitudes cognitives dans le Retard global de développement, des habiletés motrices dans le Trouble du développement de la coordination, des habiletés sociorelationnelles dans le Trouble précoce atypique du spectre de l'autisme, des capacités de régulation émotionnelle dans plusieurs troubles anxieux, etc. Le niveau de développement doit également être pris en compte dans la réflexion engagée sur l'Axe 2 car il fait clairement partie des caractéristiques de l'enfant qui contribuent aux formats relationnels observés (cf. Tableau 2). Le niveau de développement est considéré dans l'Axe 3, par exemple dans l'examen des effets de certains syndromes ou de complications périnatales. Enfin, il est également considéré dans l'Axe 4 en tant qu'il module la sensibilité de l'enfant aux facteurs de stress psychosociaux. 
L'Axe 5 a donc pour fonction d'aider le clinicien à organiser le processus d'évaluation des compétences développementales. Dans cet objectif, la nouvelle édition met à disposition en Annexe A un recensement, âge par âge (de 3 mois à 60 mois) des compétences spécifiques habituellement observées dans chacun des cinq registres considérés: développement émotionnel, socio-relationnel, communicatif, cognitif et psychomoteur. Un tableau de synthèse de l'évaluation des compétences peut ensuite être renseigné pour faire émerger une représentation d'ensemble du profil de fonctionnement actuel.

\section{Conclusion}

L'examen des invariants et des évolutions du système diagnostique proposé par la Classification 0-5 ans met en lumière l'intention de ses auteurs et l'intérêt de l'instrument. Cette nouvelle édition ne vise pas simplement à une mise à jour des catégories nosographiques dans le domaine de la santé mentale du jeune enfant et du développement. Sa philosophie générale et les améliorations apportées à son organisation multiaxiale concourent à en faire un outil au service du processus d'élaboration clinique pour mieux comprendre les difficultés d'un jeune enfant au sein de sa famille et orienter les stratégies d'aide que les équipes de professionnels de la petite enfance sont susceptibles de mettre en œuvre. Nous espérons donc que la publication de l'édition française de la Classification 0-5 ans par Médecine et Hygiène, pourra constituer, pour ces équipes de professionnels, une opportunité fructueuse.

\section{Références}

Guedeney, A., \& Le Foll, J. (sous presse). Classification diagnostique 0-3 ans (DC 0-3R, 2005) et sa révision (DC 0-5, 2016). EMC - Psychiatrie/Pédopsychiatrie.

Guedeney A, Dubois de Bodinat L. Classification diagnostique 0-3 ans (DC 0-3, 1994) et sa révision (DC 0-3R, 2005). EMC Psychiatrie (Elsevier Masson, Paris). 2009. 37200(140): 1-6

Guedeney N, Guedeney A, Rabouam C, Mintz AS, Danon G, Morales Huet M, \& Jacquemin F. The Zero To Three diagnostic classification: A contribution to the validation of this classification from a sample of 85 under three. Infant Mental Health Journal. 2003. 24, 4:313-336.

Perret P. L'édition révisée de la classification diagnostique 0-3ans et sa traduction française. Devenir.2009; $5:$ p. 7-13.

Zeanah CH, Carter A, Cohen J. et al. DC 0-3 to DC 0-3R to DC 0-5: A new edition. Zero to Three. 2015. 35, 63-66. 
Zero to Three (2016). DC: 0-5. Diagnostic Classification of mental health and developmental disorders of infancy and early childhood. Washington: Zero To Three Press. 